\title{
VOLUMES OF $n$-SIMPLICES WITH VERTICES ON A POLYNOMIAL SPACE CURVE
}

\begin{abstract}
In this paper, we give a formula for the area of the triangle formed by the vertices that live on a given polynomial, and we generalize this formula to the volumes of $n$-simplices with vertices on a polynomial space curve. To prove these results, we use induction arguments and a well known identity for complete symmetric polynomials.
\end{abstract}

Given a number $x_{1} \in \mathbb{R}$ and a parabola $p(x)=x^{2}+b x+c$ defined over $\mathbb{R}$, triangles with vertices $\left(x_{1}, p\left(x_{1}\right)\right),\left(x_{1}+1, p\left(x_{1}+1\right)\right)$ and $\left(x_{1}+2, p\left(x_{1}+2\right)\right)$ have unit area whatever the values of $b, c$ and $x_{1}$. Figure 1 shows an example.

Starting with this observation, we provide answers to the following questions:

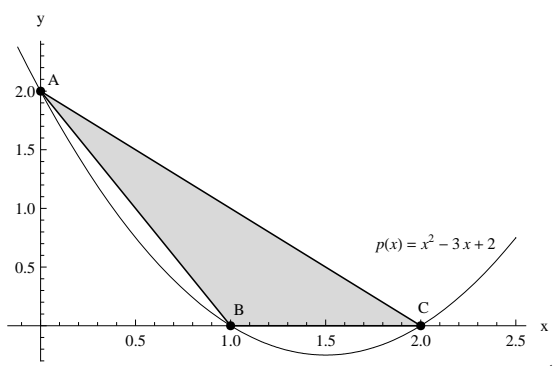

Figure 1. Triangle with vertices $(0,2)$, $(1,0)$ and $(2,0)$ living on the parabola $p(x)=x^{2}-3 x+2$.

Question 1. Let $p(t)=\sum_{k=0}^{N} a_{k} t^{k}$ be polynomial of degree $N \geq 2$, i.e. $a_{N} \neq 0$, with real coefficients. For any given three real numbers $x_{1}, x_{2}, x_{3}$, we consider the triangle $A B C$ formed by the vertices $A=\left(x_{1}, p\left(x_{1}\right)\right), B=\left(x_{2}, p\left(x_{2}\right)\right), C=\left(x_{3}, p\left(x_{3}\right)\right)$. If any two of $x_{1}, x_{2}, x_{3}$ are equal to each other then, clearly the area of $A B C$ is 0 .

Can we obtain a formula in factorized form for the area of $A B C$ so that we can see the effects of the polynomial $p(t)$, and $x_{1}, x_{2}$, and $x_{3}$ ? If so, can we generalize the result to higher dimensions?

The answers we provide for this question are Theorem 4 and Theorem 8 ,

Question 2. The following identity is well known.

$$
x^{m}-y^{m}=(x-y)\left(x^{m-1}+x^{m-2} y+\cdots+x y^{m-2}+y^{m-1}\right), \quad \text { for any } 2 \leq m \in \mathbb{N} .
$$

What further generalizations of this identity are there? For example, what would be the analogues identity in $n \geq 3$ variables?

Proposition 3 and Theorem 7 answer question 2 .

Question 3. For any given $d \geq 0$ and $n \geq 2$, are there any "short" algebraic expressions for the complete symmetric polynomial of degree $d$ in $n$ variables?

Question 3 is closely related to question 2 . Theorem 5 provides an answer for question 3 .

We start by considering question 2. First, we notice the appearance of all monomials of degree $m$, in $x$ and $y$, in Equation (11). This suggests that any generalization of (1) to more variables should involve the complete symmetric polynomials. Indeed this is the case, as we shall see. Let's recall their definition and establish some notation.

For any $0 \leq d \in \mathbb{N}$ and $1 \leq n \in \mathbb{N}$, the complete symmetric polynomial of degree $d$ in $n$ variables is defined to be the sum of all monomials of degree $d$ in $n$ variables. For

Key words and phrases. Complete Symmetric Polynomials, Disciminant, Alternant, Triangle Area, volumes of $n$-parallelpipeds, volumes of $n$-simplices. 
variables $x_{1}, x_{2}, \ldots, x_{n}$, we set $h_{d}\left(x_{1}, x_{2}, \ldots, x_{n}\right)=\sum_{d_{1}+d_{2}+\cdots+d_{n}=d} x_{1}^{d_{1}} x_{2}^{d_{2}} \ldots x_{n}^{d_{n}}$, where $d_{i}$ is a nonnegative integer for any $i=1,2, \ldots, n$. By convention, we set $h_{0}\left(x_{1}, x_{2}, \ldots, x_{n}\right)=1$ for any $n \geq 1$. See [1, Chapter 1] for a discussion of basic properties of complete symmetric polynomials.

We can rewrite Equation (11) as $x_{1}^{m}-x_{2}^{m}=\left(x_{1}-x_{2}\right) h_{m-1}\left(x_{1}, x_{2}\right)$ for $m \geq 1$.

Lemma 1. For $1 \leq s \in \mathbb{N}$ and $2 \leq n \in \mathbb{N}, h_{s}\left(x_{1}, x_{2}, \ldots, x_{n}\right)=\sum_{j=1}^{n} x_{j} h_{s-1}\left(x_{j}, x_{j+1}, \ldots, x_{n}\right)$.

Proof. The proof follows by grouping the monomials as the ones that are divisible by $x_{1}$, the ones that are not divisible by $x_{1}$ but divisible by $x_{2}$, the ones that are not divisible by $x_{1}$ and $x_{2}$ but divisible by $x_{3}$, and so on.

Lemma 2. For $2 \leq m \in \mathbb{N}$,

$$
h_{m}\left(x_{1}, x_{2}, x_{3}\right)=\left(x_{1}+x_{2}\right) h_{m-1}\left(x_{1}, x_{2}, x_{3}\right)-x_{1} x_{2} h_{m-2}\left(x_{1}, x_{2}, x_{3}\right)+x_{3}^{m} .
$$

Proof. We use Lemma 1 to derive the result.

$$
\begin{aligned}
& h_{m}\left(x_{1}, x_{2}, x_{3}\right) \\
& =x_{1} h_{m-1}\left(x_{1}, x_{2}, x_{3}\right)+x_{2} h_{m-1}\left(x_{2}, x_{3}\right)+x_{3}^{m}, \quad \text { by Lemma } 1 \text { with } s=m \text { and } n=3 \\
& =x_{1} h_{m-1}\left(x_{1}, x_{2}, x_{3}\right)+x_{2}\left(x_{2} h_{m-2}\left(x_{2}, x_{3}\right)+x_{3}^{m-1}\right)+x_{3}^{m}, \quad \text { by Lemma } 1 \text { with } s=m-1 \\
& \text { and } n=2 \text {. Then, Lemma } 1 \text { with } s=m-1 \text { and } n=3 \text { gives } \\
& =x_{1} h_{m-1}\left(x_{1}, x_{2}, x_{3}\right)+x_{2}\left(h_{m-1}\left(x_{1}, x_{2}, x_{3}\right)-x_{1} h_{m-2}\left(x_{1}, x_{2}, x_{3}\right)-x_{3}^{m-1}+x_{3}^{m-1}\right)+x_{3}^{m} \\
& =\left(x_{1}+x_{2}\right) h_{m-1}\left(x_{1}, x_{2}, x_{3}\right)-x_{1} x_{2} h_{m-2}\left(x_{1}, x_{2}, x_{3}\right)+x_{3}^{m} .
\end{aligned}
$$

The following proposition gives a partial answer to question 2. It is the generalization of Equation (1) to three variables.

Proposition 3. For $1 \leq m \in \mathbb{N}$ and variables $x_{1}, x_{2}, x_{3}$,

$$
\left(x_{3}-x_{2}\right) x_{1}^{m+1}+\left(x_{1}-x_{3}\right) x_{2}^{m+1}+\left(x_{2}-x_{1}\right) x_{3}^{m+1}=\left(x_{3}-x_{2}\right)\left(x_{3}-x_{1}\right)\left(x_{2}-x_{1}\right) h_{m-1}\left(x_{1}, x_{2}, x_{3}\right) .
$$

Proof. The proof is by induction on $m$. Since $h_{0}\left(x_{1}, x_{2}, x_{3}\right)=1$ and $h_{1}\left(x_{1}, x_{2}, x_{3}\right)=x_{1}+$ $x_{2}+x_{3}$, the equalities for $m=1$ and $m=2$ can be verified by a simple algebra.

Suppose that the equality is true for all $i$ such that $2 \leq i \leq m$. Then, in particular, for the cases $i=m$ and $i=m-1$ we have

For $i=m+1$, by Lemma 2 ,

$$
\begin{aligned}
& \left(x_{3}-x_{2}\right)\left(x_{3}-x_{1}\right)\left(x_{2}-x_{1}\right) h_{m}\left(x_{1}, x_{2}, x_{3}\right) \\
& =\left(x_{3}-x_{2}\right)\left(x_{3}-x_{1}\right)\left(x_{2}-x_{1}\right)\left(\left(x_{1}+x_{2}\right) h_{m-1}\left(x_{1}, x_{2}, x_{3}\right)-x_{1} x_{2} h_{m-2}\left(x_{1}, x_{2}, x_{3}\right)+x_{3}^{m}\right) \\
& =\left(x_{3}-x_{2}\right) x_{1}^{m+2}+\left(x_{1}-x_{3}\right) x_{2}^{m+2}+\left(x_{2}-x_{1}\right) x_{3}^{m+2} .
\end{aligned}
$$

The last equality is by Equation (2) and Equation (3). 
Before answering question 2 for $n \geq 4$ variables, we answer question 1 as an application of Proposition 3 .

Let $p(t), x_{1}, x_{2}, x_{3}$, and the triangle $A B C$ be as given in question 1. Then the signed area $A\left(x_{1}, x_{2}, x_{3}\right)$ of the triangle $A B C$ is given by $\frac{1}{2} \operatorname{det}(\mathbf{M})$, where $\mathbf{M}=\left(\begin{array}{lll}1 & x_{1} & p\left(x_{1}\right) \\ 1 & x_{2} & p\left(x_{2}\right) \\ 1 & x_{3} & p\left(x_{3}\right)\end{array}\right)$. Thus the area of $A B C$ is $\left|A\left(x_{1}, x_{2}, x_{3}\right)\right|$ and we have

$$
A\left(x_{1}, x_{2}, x_{3}\right)=\frac{1}{2}\left(\left(x_{3}-x_{2}\right) p\left(x_{1}\right)-\left(x_{3}-x_{1}\right) p\left(x_{2}\right)+\left(x_{2}-x_{1}\right) p\left(x_{3}\right)\right)
$$

(See [2, Page 1], for the relation between this area and the convexity of $p(t)$.) We note that $\operatorname{det}(\mathbf{M})$ is a particular example of an alternant, a definition first used by Sylvester [3, Page 322] for determinants of matrices such that $i$-th row of $\mathbf{M}$ are functions of variable $x_{i}$ and same functions are used for each row. As mentioned in the article [3], differences of the variables divide $A\left(x_{1}, x_{2}, x_{3}\right)$. By the following theorem, which uses Proposition 3 , we express $A\left(x_{1}, x_{2}, x_{3}\right)$ in a factorized form.

Theorem 4. Let the polynomial $p(t)=a_{0}+a_{1} t+\cdots+a_{N} t^{N}$ and the triangle $A B C$ be as above, then

$$
\left|A\left(x_{1}, x_{2}, x_{3}\right)\right|=\frac{1}{2}\left|x_{1}-x_{2}\right|\left|x_{1}-x_{3}\right|\left|x_{2}-x_{3}\right|\left|\sum_{k=2}^{N} a_{k} h_{k-2}\left(x_{1}, x_{2}, x_{3}\right)\right| .
$$

Proof. The proof is given by induction on $N$, the degree of the polynomial $p(t)$. Without loss of generality, suppose that $x_{1} \leq x_{2} \leq x_{3}$. It is enough to prove that

$$
A\left(x_{1}, x_{2}, x_{3}\right)=\frac{1}{2}\left(x_{2}-x_{1}\right)\left(x_{3}-x_{1}\right)\left(x_{3}-x_{2}\right) \sum_{k=2}^{N} a_{k} h_{k-2}\left(x_{1}, x_{2}, x_{3}\right) .
$$

If $N=2, A\left(x_{1}, x_{2}, x_{3}\right)$ simplifies to $\frac{1}{2}\left(x_{2}-x_{1}\right)\left(x_{3}-x_{1}\right)\left(x_{3}-x_{2}\right) a_{2}$, which is the desired form since $h_{0}\left(x_{1}, x_{2}, x_{3}\right)=1$.

Assume that for some $N \geq 2$, Equation (15) holds for all polynomials of degree at most $N$. Then, given a polynomial $P(t)$ of degree $N+1$, we can write $P(t)=a_{N+1} t^{N+1}+p(t)$ where $a_{N+1} \neq 0$ and $p(t)$ has degree at most $N$. We then have

$$
\begin{aligned}
A\left(x_{1}, x_{2}, x_{3}\right)= & \frac{1}{2}\left(\left(x_{3}-x_{2}\right)\left(a_{N+1} x_{1}^{N+1}+p\left(x_{1}\right)\right)-\left(x_{3}-x_{1}\right)\left(a_{N+1} x_{2}^{N+1}+p\left(x_{2}\right)\right)\right. \\
& \left.\quad+\left(x_{2}-x_{1}\right)\left(a_{N+1} x_{3}^{N+1}+p\left(x_{3}\right)\right)\right), \quad \text { by Equation (44). } \\
= & \frac{1}{2}\left(\left(x_{3}-x_{2}\right) p\left(x_{1}\right)-\left(x_{3}-x_{1}\right) p\left(x_{2}\right)+\left(x_{2}-x_{1}\right) p\left(x_{3}\right)\right) \\
& \quad+\frac{1}{2} a_{N+1}\left(\left(x_{3}-x_{2}\right) x_{1}^{N+1}-\left(x_{3}-x_{1}\right) x_{2}^{N+1}+\left(x_{2}-x_{1}\right) x_{3}^{N+1}\right) \\
= & \frac{1}{2}\left(x_{2}-x_{1}\right)\left(x_{3}-x_{1}\right)\left(x_{3}-x_{2}\right) \sum_{k=2}^{N} a_{k} h_{k-2}\left(x_{1}, x_{2}, x_{3}\right)+\frac{1}{2} a_{N+1}\left(\left(x_{3}-x_{2}\right) x_{1}^{N+1}\right. \\
& \left.\quad-\left(x_{3}-x_{1}\right) x_{2}^{N+1}+\left(x_{2}-x_{1}\right) x_{3}^{N+1}\right), \quad \text { by induction. }
\end{aligned}
$$




$$
=\frac{1}{2}\left(x_{2}-x_{1}\right)\left(x_{3}-x_{1}\right)\left(x_{3}-x_{2}\right) \sum_{k=2}^{N+1} a_{k} h_{k-2}\left(x_{1}, x_{2}, x_{3}\right), \quad \text { by Proposition } 3 \text {. }
$$

We answer question 3 by the following well-known identity for complete symmetric homogeneous polynomials (see [4, Ex 7.4, p. 450 and p. 490]). We provide an elementary proof which relies on induction arguments.

Theorem 5. For $1 \leq m \in \mathbb{N}, 2 \leq n \in \mathbb{N}$, and distinct variables $x_{1}, x_{2}, \ldots x_{n}$,

$$
\sum_{i=1}^{n} \frac{x_{i}^{n+m-1}}{\prod_{\substack{j=1 \\ j \neq i}}^{n}\left(x_{i}-x_{j}\right)}=h_{m}\left(x_{1}, x_{2}, \ldots, x_{n}\right) .
$$

Proof. We obtain the result by induction on $K=n+m \geq 3$. The cases $K=3$ and $K=4$ follows from Equation (1) and Equation (3), respectively. Suppose $K>4$.

$$
\begin{aligned}
h_{m}\left(x_{1}, x_{2}, \ldots, x_{n}\right) & =h_{m}\left(x_{1}, x_{2}, \ldots, x_{n-1}\right)+x_{n} \cdot h_{m-1}\left(x_{1}, x_{2}, \ldots, x_{n}\right), \quad \text { by grouping } \\
& =\sum_{i=1}^{n-1} \frac{x_{i}^{n+m-2}}{\prod_{\substack{n=1 \\
j \neq i}}^{n-1}\left(x_{i}-x_{j}\right)}+x_{n} \sum_{i=1}^{n} \frac{x_{i}^{n+m-2}}{\prod_{\substack{j=1 \\
j \neq i}}^{n}\left(x_{i}-x_{j}\right)}, \quad \text { by induction } \\
& =\sum_{i=1}^{n-1} \frac{x_{i}^{n+m-2}\left(x_{i}-x_{n}\right)}{\prod_{\substack{j=1 \\
j \neq i}}^{n}\left(x_{i}-x_{j}\right)}+\sum_{i=1}^{n-1} x_{n} \frac{x_{i}^{n+m-2}}{\prod_{\substack{j=1 \\
j \neq i}}^{n}\left(x_{i}-x_{j}\right)}+\frac{x_{n}^{n+m-1}}{\prod_{\substack{j=1 \\
j \neq i}}^{n}\left(x_{i}-x_{j}\right)} .
\end{aligned}
$$

This gives the result.

If $n<m$, Theorem 5 can be used to compute $h_{m}\left(x_{1}, x_{2}, \ldots, x_{n}\right)$ effectively. A result closely related to Theorem 5 is the following theorem:

Theorem 6. Let $2 \leq n \in \mathbb{N}$, and let $x_{1}, x_{2}, \ldots, x_{n}$ be distinct variables. Then for any $0 \leq k \leq n-2$, we have $\sum_{i=1}^{n} \frac{x_{i}^{k}}{\prod_{\substack{j=1 \\ j \neq i}}^{n}\left(x_{i}-x_{j}\right)}=0$.

Proof. Case 1: $k=n-2$. For $n=2$, we have $\frac{1}{x_{1}-x_{2}}+\frac{1}{x_{2}-x_{1}}=0$. Let $n>2$, and let $k=n-2$. Lagrange interpolation formula applied to the polynomial $q\left(x_{n}\right)=-x_{n}^{k}$ gives

$$
-x_{n}^{k}=\sum_{i=1}^{n-1} \frac{-x_{i}^{k} \cdot \prod_{\substack{j=1 \\ j \neq i}}^{n-1}\left(x_{n}-x_{j}\right)}{\prod_{\substack{j=1 \\ j \neq i}}^{n-1}\left(x_{i}-x_{j}\right)}=\sum_{i=1}^{n-1} \frac{x_{i}^{k} \cdot \prod_{j=1}^{n-1}\left(x_{n}-x_{j}\right)}{\prod_{\substack{j=1 \\ j \neq i}}^{n}\left(x_{i}-x_{j}\right)}=\prod_{j=1}^{n-1}\left(x_{n}-x_{j}\right) \sum_{i=1}^{n-1} \frac{x_{i}^{k}}{\prod_{\substack{j=1 \\ j \neq i}}^{n}\left(x_{i}-x_{j}\right)} .
$$

This completes the proof in this case.

Case 2: $0 \leq k=n-3$. We have

$$
\sum_{i=1}^{n-1} \frac{x_{i}^{n-3}}{\prod_{\substack{j=1 \\ j \neq i}}^{n-1}\left(x_{i}-x_{j}\right)}=\sum_{i=1}^{n-1} \frac{x_{i}^{n-3}\left(x_{i}-x_{n}\right)}{\prod_{\substack{j=1 \\ j \neq i}}^{n}\left(x_{i}-x_{j}\right)}=\sum_{i=1}^{n-1} \frac{x_{i}^{n-2}}{\prod_{\substack{j=1 \\ j \neq i}}^{n}\left(x_{i}-x_{j}\right)}-x_{n} \sum_{i=1}^{n-1} \frac{x_{i}^{n-3}}{\prod_{\substack{j=1 \\ j \neq i}}^{n}\left(x_{i}-x_{j}\right)} .
$$

Equivalently, $\sum_{i=1}^{n-1} \frac{x_{i}^{n-3}}{\prod_{\substack{j=1 \\ j \neq i}}^{n-1}\left(x_{i}-x_{j}\right)}=\sum_{i=1}^{n} \frac{x_{i}^{n-2}}{\prod_{\substack{j=1 \\ j \neq i}}^{n}\left(x_{i}-x_{j}\right)}-x_{n} \sum_{i=1}^{n} \frac{x_{i}^{n-3}}{\prod_{\substack{j=1 \\ j \neq i}}^{n}\left(x_{i}-x_{j}\right)}$. Note that case 
1 with $n-1$ and $n$ implies $\sum_{i=1}^{n-1} \frac{x_{i}^{n-3}}{\prod_{\substack{j=1 \\ j \neq i}}^{n-1}\left(x_{i}-x_{j}\right)}=0=\sum_{i=1}^{n} \frac{x_{i}^{n-2}}{\prod_{\substack{j=1 \\ j \neq i}}^{n}\left(x_{i}-x_{j}\right)}$. This completes the proof in this case.

Following the strategy of case 2 successively, one can prove the result for each $k$ with $0 \leq k \leq n-4$.

We now generalize Proposition 3 and Theorem 4 to an arbitrary number of variables. We first note that discriminants of the variables appear in both Equation (11) and Proposition 3 ,

A difference product of the variables $x_{1}, x_{2}, \ldots, x_{n}$ is the following Vandermonde determinant: $d\left(x_{1}, x_{2}, \ldots, x_{n}\right)=\operatorname{det}(\mathbf{D})$, where $\mathbf{D}=\left(\begin{array}{ccccc}1 & x_{1} & x_{1}^{2} & \ldots & x_{1}^{n-1} \\ 1 & x_{2} & x_{2}^{2} & \ldots & x_{2}^{n-1} \\ \ldots & \ldots & \ldots & \ldots & \ldots \\ 1 & x_{n} & x_{n}^{2} & \ldots & x_{n}^{n-1}\end{array}\right)_{n \times n}$. It was shown by Cauchy in 1812 that

$$
d\left(x_{1}, x_{2}, \ldots, x_{n}\right)=\prod_{1 \leq i<j \leq n}\left(x_{j}-x_{i}\right)
$$

The following theorem is the answer for question 2 for arbitrary number of variables:

Theorem 7. For $0 \leq m \in \mathbb{N}, 2 \leq n \in \mathbb{N}$, and distinct variables $x_{1}, x_{2}, \ldots, x_{n}$, we have

$$
\sum_{k=1}^{n}(-1)^{n-k} x_{k}^{n+m-1} d\left(x_{1}, x_{2}, \ldots, x_{k-1}, x_{k+1}, \ldots, x_{n}\right)=d\left(x_{1}, x_{2}, \ldots, x_{n}\right) h_{m}\left(x_{1}, x_{2}, \ldots, x_{n}\right) .
$$

Proof. Using Equation ([6) $), d\left(x_{1}, x_{2}, \ldots, x_{k-1}, x_{k+1}, \ldots, x_{n}\right)=\prod_{\substack{1 \leq i<j \leq n \\ i \neq k \neq j}}^{n}\left(x_{j}-x_{i}\right)$. Then the result follows from Theorem 5 ,

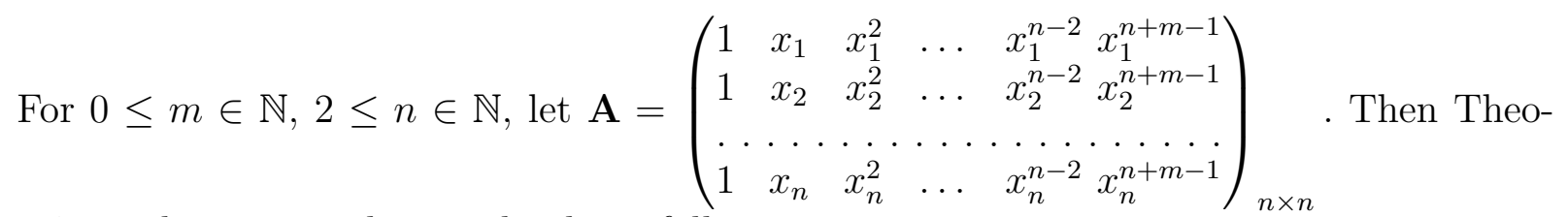
rem 7 can be expressed equivalently as follows:

$$
\operatorname{det}(\mathbf{A})=d\left(x_{1}, x_{2}, \ldots, x_{n}\right) h_{m}\left(x_{1}, x_{2}, \ldots, x_{n}\right) .
$$

We now generalize Theorem 4 to an arbitrary number of variables. Let the polynomial $p(t)$

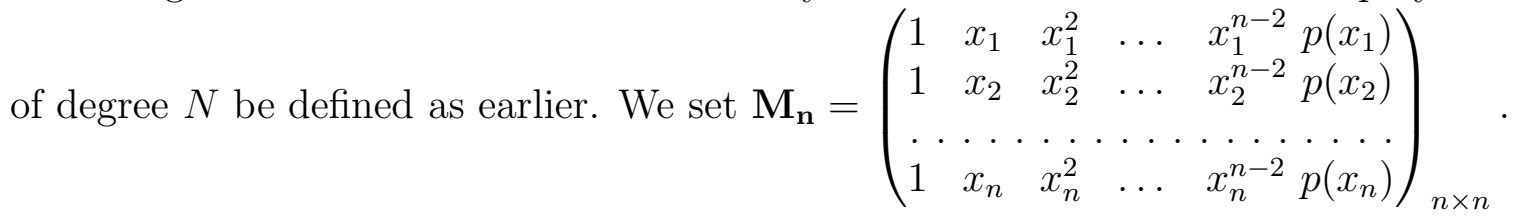

Remark. The volume of $n$-parallelpipeds generated by the column vectors of $\mathbf{M}_{\mathbf{n}}$ is $\left|\operatorname{det}\left(\mathbf{M}_{\mathbf{n}}\right)\right|$, and the volumes of the $n$-simplices generated by the column vectors of $\mathbf{M}_{\mathbf{n}}$ is $\frac{1}{n !}\left|\operatorname{det}\left(\mathbf{M}_{\mathbf{n}}\right)\right|$.

Theorem 8. Let $2 \leq n \in \mathbb{N}$. If $N \leq n-2$, then $\operatorname{det}\left(\mathbf{M}_{\mathbf{n}}\right)=0$. If $n-1 \leq N \in \mathbb{N}$,

$$
\operatorname{det}\left(\mathbf{M}_{\mathbf{n}}\right)=d\left(x_{1}, x_{2}, \ldots, x_{n}\right) \sum_{k=n-1}^{N} a_{k} h_{k-n+1}\left(x_{1}, x_{2}, \ldots, x_{n}\right) \text {. }
$$


Proof. The proof is given by induction on $N$, the degree of the polynomial $p(t)$ (as in the proof of Theorem 4), and by using Equation (7). The details are left as an exercise to the reader.

Acknowledgements. I would like to thank Dr. Robert Rumely for useful discussions concerning this paper.

\section{REFERENCES}

[1] I.G. Macdonald, Symmetric Functions and Hall Polynomials, 2nd edition, Clarendon Press, Oxford 1998.

[2] D.S. Mitrinovic, J.E. Pecaric and A.M. Fink, Classical and New Inequalities in Analysis, Kluwer Academic Publishers, Dordrect/Boston/London.

[3] Muir Thomas (revised by H.Metzler, William), A Treatise on the Theory of Determinants, Dover Publications Inc, New York, 1960.

[4] Richard P. Stanley, Enumerative Combinatorics, Cambridge University Press, 1999. 